\title{
Peer victimisation during adolescence and its impact on depression in early adulthood: prospective cohort study in the United Kingdom
}

\author{
Lucy Bowes, ${ }^{1}$ Carol Joinson, ${ }^{2}$ Dieter Wolke, ${ }^{3}$ Glyn Lewis ${ }^{4}$
}

1Department of Experimental

Psychology, University of

Oxford, Oxford OX1 3UD, UK

${ }^{2}$ Centre for Academic Mental

Health, School of Social and

Community Medicine, University

of Bristol, Bristol, UK

${ }^{3}$ Department of Psychology and

Division of Mental Health and

Wellbeing, University of

Warwick, Coventry, UK

${ }^{4}$ Division of Psychiatry, Faculty

of Brain Sciences, University

College London, London, UK

Correspondence to: L Bowes lucy.bowes@psy.ox.ac.uk

Cite this as: BMJ 2015;350:h2469 doi: 10.1136/bmj.h2469

Accepted: 30 March 2015

\author{
ABSTRACT \\ OBJECTIVE \\ To investigate the strength of the association between \\ victimisation by peers at age 13 years and depression \\ at 18 years.
}

DESIGN

Longitudinal observational study.

SETTING

Avon Longitudinal Study of Parents and Children, a UK community based birth cohort.

\section{PARTICIPANTS}

6719 participants who reported on peer victimisation at age 13 years.

\section{MAIN OUTCOME MEASURES}

Depression defined according to international classification of diseases, 10th revision (ICD-10) criteria, assessed using the clinical interview schedule-revised during clinic assessments with participants when they were aged 18 years. 3898 participants had data on both victimisation by peers at age 13 years and depression at age 18 years.

RESULTS

Of the 683 participants who reported frequent victimisation at age 13 years, 101 (14.8\%) were depressed according to ICD-10 criteria at 18 years; of the 1446 participants reporting some victimisation at age 13 years, $103(7.1 \%)$ were depressed at age 18 years; and of the 1769 participants reporting no victimisation at age 13 years, 98 (5.5\%) were depressed at age 18 years. Compared with children who were not victimised those who were frequently victimised by peers had over a twofold increase in odds of depression (odds ratio 2.96, 95\% confidence interval 2.21 to $3.97, \mathrm{P}<0.001)$. This association was slightly reduced when adjusting for confounders (2.32, 1.49 to $3.63, \mathrm{P}<0.001)$. The population attributable

\section{WHAT IS ALREADY KNOWN ON THIS TOPIC}

Numerous studies have shown that victimisation by peers is associated with an increased risk of internalising problems in childhood

It remains unclear whether peer victimisation contributes to the public health burden of depression that mainly manifests in adulthood because of limitations in previous studies

\section{WHAT THIS STUDY ADDS}

Our observational findings suggest that approximately $29 \%$ of the burden of depression at age 18 years could be attributed to victimisation by peers in adolescence if this relation were causal

This study used a well validated measure of peer victimisation and was able to adjust for several factors that might have caused both victimisation and depression fraction suggested that $29.2 \%$ ( $95 \%$ confidence interval $10.9 \%$ to $43.7 \%$ ) of depression at age 18 years could be explained by peer victimisation if this were a causal relation.

\section{CONCLUSION}

When using observational data it is impossible to be certain that associations are causal. However, our results are consistent with the hypothesis that victimisation by peers in adolescence is associated with an increase in the risk of developing depression as an adult.

\section{Introduction}

Depression is a leading contributor to the global burden of disease. ${ }^{1}$ The incidence and prevalence of depression increases rapidly from childhood to early adulthood, and by age 18 years the prevalence is similar to that in adults. $^{2}$ This has led to suggestions that school based preventive programmes could help to reduce the burden of depression. However, the results of cognitive behaviour programmes in schools have been disappointing. ${ }^{3}$

Adolescents spend more time with their peers than do children and adults, and peers are used as primary sources for social comparison and appraisal. ${ }^{4}$ Victimisation by peers or "bullying" has been proposed as one potentially modifiable risk factor for depression. Cross sectional studies report correlations between peer victimisation and clinical depression, ${ }^{56}$ whereas adults with depression are more likely to retrospectively report peer victimisation as a child or adolescent $^{7}$; however, these results could be due to recall bias or reverse causality. Previous longitudinal studies have mainly examined outcomes such as internalising symptoms in childhood or adolescence ${ }^{8-10}$ rather than studying diagnoses of depression at older ages, which has more relevance for the population burden of depression that occurs mainly in adults of working age. ${ }^{11} 12$

We are only aware of a small number of longitudinal studies that have prospectively investigated victimisation in relation to depression meeting diagnostic criteria in late adolescence or adulthood. Some of these did not adjust for key confounders such as baseline depressive symptoms ${ }^{13}$ or behavioural problems, ${ }^{14}$ which might themselves have led to both victimisation and later depression. Others ${ }^{15} 16$ have only found an association for children who were both victims and perpetrators of bullying. Perpetrators also display high levels of conduct problems and reactive aggression, which are themselves associated with later depression ${ }^{17} 18$ and may confound the association. Others have not used a 
well validated measure of bullying, relying on reports from single items. ${ }^{14-1619}$ Given the limitations of previous studies, it still remains unclear whether peer victimisation during adolescence contributes to the public health burden of depression that mostly manifests in adulthood.

Using data from over 3700 families from the Avon Longitudinal Study of Parents and Children (ALSPAC) cohort in the United Kingdom, we investigated the strength of the association between being victimised by peers in early adolescence and the emergence of depression at age 18 years, while taking account of several confounders, including previous victimisation in childhood and mental health problems. All participants with data on peer victimisation at age 13 years and at least one assessment of depressive symptoms were eligible to be included in the study. Our hypothesis was that peer victimisation during adolescence would be associated with an increased risk of developing depression at age 18 years. We also estimated the incidence of depression that might be attributable to peer victimisation in adolescence if this relation was causal.

\section{Methods \\ Sample}

The sample comprised participants from the ALSPAC cohort (www.alspac.bris.ac.uk) ${ }^{20}$; ALSPAC is a transgenerational prospective observational study investigating a wide range of influences on the health and development of children. All pregnant women resident in the former Avon Health Authority in south west England, with an estimated date of delivery between 1 April 1991 and 31 December 1992, were eligible to take part. Women were recruited as early as possible in their pregnancy, resulting in a cohort of 14541 pregnancies and 13988 children alive at 12 months of age. When the oldest children were aged 7 years, we attempted to increase the size of the initial sample with eligible cases that did not join the cohort at the outset. The phases of enrolment are described in more detail in the cohort profile paper. ${ }^{21}$ In the current study we use data from the subsample of ALSPAC who attended the most recent research clinic for the children.

\section{Depression}

Participants completed a self administered computerised version of the clinical interview schedule-revised ${ }^{22}$ at the 18 year research clinic (mean age 17 years 10 months). This schedule enables diagnoses for common mental disorders to be derived from the international statistical classification of diseases, 10th revision (ICD10). Participants were asked about symptoms of depression in the past one week to one month (depending on the symptom). In addition, participants were asked the question: "How long have you been feeling sad, miserable, or depressed, or unable to enjoy or take an interest in things you have described?," responding "less than two weeks” ( $n=507)$, “two weeks to six months” $(n=525)$, "six months to one year" ( $\mathrm{n}=194)$, "one to two years" $(n=100)$, or "two or more years" $(n=87)$.

\section{Peer victimisation}

We assessed peer victimisation through self report using a modified version of the bullying and friendship interview schedule. ${ }^{23}$ The items are listed in table 1. Participants were asked whether they had experienced nine different types of peer victimisation in the past six months, responding "no," "yes sometimes" (<4 times), "yes repeatedly" ( $\geq 4$ times), or "yes very frequently" (at least once per week). The items related to both relational and overt victimisation (for example, exclusion by peers, lies or nasty things said about them, personal belongings taken, threatened or blackmailed, hit or beaten up). In preliminary analyses we found that scores for relational and overt victimisation were strongly correlated $(r=0.61)$. Furthermore, we did not believe that separating the two broad types of victimisation would substantially add to the policy implications of our study, and so we used an overall measure of peer victimisation.

The victimisation variable was created from the sum of all questions relating to victimisation. Children scored 0 if they had never been bullied. The range of scores was 0-25 (mean 1.85, SD 2.78) and Cronbach's $\alpha$ was 0.73 . To investigate a possible dose-response relation between victimisation and depression, we also created a three level ordinal variable for victimisation. ${ }^{24}$ Children who were never victimised $(n=3090)$ scored 0 , children who were occasionally victimised $(n=2430)$ scored 1-3 and were coded as 1, and children who were frequently victimised $(n=1199)$ scored 4 or more and were coded as 2 .

Peer victimisation was also reported by mothers when their children were 12 years of age. During questionnaire assessments the mothers were asked whether their offspring was often picked on or bullied by other children, ${ }^{25}$ responding not true $(n=5522,80.5 \%)$, somewhat true $(n=1108,16.2 \%)$, or certainly true $(n=229$, $3.3 \%)$. The inter-rater agreement between mother and self reports of peer victimisation was low $(\kappa=0.10)$, similar to that in other studies. ${ }^{26}$

\section{Peer victimisation at ages 8 and 10}

Peer victimisation was also assessed through interviews with the children at ages 8 and 10 years, using the same version of the bullying and friendship interview schedule. Our measure of victimisation at each time point was created using the same procedure as described for age 13 years. Cronbach's $\alpha$ was 0.77 and 0.70 , respectively.

The polychoric correlations for peer victimisation at age 13 years was 0.29 (SE 0.02) for peer victimisation at age 8 years and 0.40 (SE 0.01) for peer victimisation at age 10 years.

\section{Confounders Individual characteristics of study children}

We assessed the children's emotional and behavioural problems using maternal reports from the strengths and difficulties questionnaire, ${ }^{25}$ when the children were aged 7 years. Concurrent depressive mood was assessed using the self reported short moods and feelings 
questionnaire, ${ }^{27}$ when the children were aged 13 years. Perpetration of concurrent bullying at age 13 was assessed using the modified version of the bullying and friendship interview schedule. ${ }^{23}$ As with the victimisation variable, we created the bullying variable from the sum of all questions relating to perpetration of bullying. Children scored 0 if they had never bullied another child (range 0-25).

\section{Family characteristics}

We adjusted for the effects of several family characteristics, including highest maternal education (dichotomised to advanced level qualifications, university degree, ordinary level qualifications versus certificate of secondary school education, vocational, none); parental occupational social class (derived as the lower of either maternal or paternal social class and dichotomised into non-manual and manual work), ${ }^{28}$ maternal depression (Edinburgh postnatal maternal depression scale ${ }^{29}$ ), and child maltreatment (no or present) when the study child was aged 7 years, assessed using maternal reports of the study children's exposure to stressful life events between 5 and 7 years of age. A score of 1 was coded if parents responded yes to any item relating to physical or sexual abuse, or reported that the study child had been put into care.

\section{Statistical analyses}

In univariable models we used logistic regression analyses to calculate odds ratios for depression at age 18 according to victimisation at age 13 (measured as a three level categorical victimisation variable and as a continuous variable). A quadratic term was used to investigate the possibility of a non-linear relation between victimisation and depression. We conducted a final multivariable model including all confounding variables. To investigate whether any effects of victimisation as an adolescent and victimisation as a child (assessed at ages 8 and 10 years) were independent of each other, we examined a series of logistic regression models, and then included all three time points of victimisation into a multivariable model.

We used the punaf command available in STATA 12 to calculate the population attributable risk and 95\% confidence interval from the final multivariable logistic regression model.

\section{Missing data}

A sample with complete data across all exposure, outcome, and confounding variables $(\mathrm{n}=2668)$ was used to investigate the main and independent effects of victimisation by peers in adolescence. In ALSPAC there is a wealth of information on sociodemographic variables and other variables that predict missingness. These data therefore make the "missing at random" assumption underlying multiple imputation much more reasonable. We could also use previous reports of depressive symptoms to impute ICD-10 depressive disorder at age 18 up to a sample size of 6472.
We used multivariate imputation by fully conditional specification using chained equations (MICE) in Stata $12 .{ }^{30}$ Multiple imputation was conducted in two stages: imputing missing confounders to give a sample size of 3898, then imputing on outcome to give a sample size of 6472 . The imputation model included 18 variables in addition to those included in the analyses that were associated with either missingness or depression at age 18 years. These included maternal age and family adversity, and sociodemographics in pregnancy and early childhood (the full list is available on request). We used Rubin rules to average the variable estimates over 60 imputed or completed datasets. ${ }^{31}$

\section{Results}

Data on victimisation by peers at age 13 years were available for 6719 participants. Of these, 3898 completed the clinical interview schedule-revised at age 18 years. In total, 2668 participants had complete data on all variables, including confounders (figure). Attrition depended on several factors, but those lost to follow-up were no more likely to have been bullied as an adolescent (odds ratio 1.00, 95\% confidence interval 0.98 to $1.02, \mathrm{P}=0.78$ ) than those with complete data.

Table 1 shows the frequencies of victimisation experiences and percentage of children who did not tell anyone at home or the teacher at school. The most commonly reported type of victimisation experience was being called nasty names or having personal belongings taken. Most teenagers (41-74\%) reported that they never told their teachers about their victimisation experiences, and

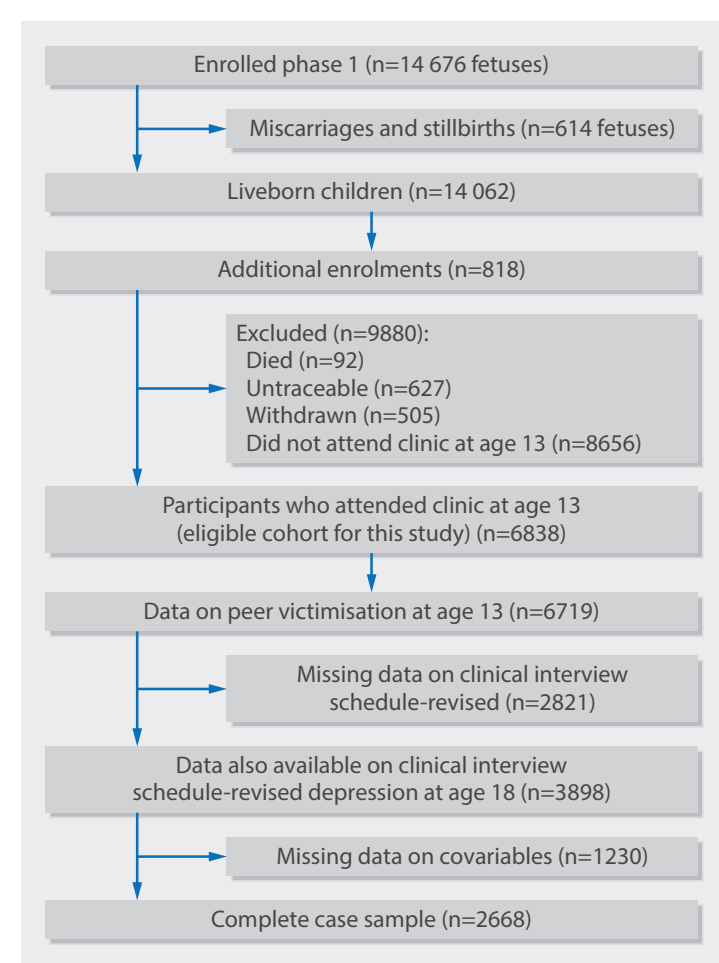

Flowchart of participants in Avon Longitudinal Study of Parents and Children 


\begin{tabular}{|c|c|c|c|c|c|c|}
\hline \multirow[b]{2}{*}{ Items } & \multicolumn{4}{|c|}{ Frequency of victimisation } & \multicolumn{2}{|c|}{$\begin{array}{l}\text { Not reporting } \\
\text { victimisation (\%) }\end{array}$} \\
\hline & Never* & $\begin{array}{l}\text { Occasional* } \\
\text { (1-3 times) }\end{array}$ & $\begin{array}{l}\text { Frequently* } \\
\text { (>4 times) }\end{array}$ & $\begin{array}{l}\text { Very frequently } \\
(>1 / \text { week) }\end{array}$ & $\begin{array}{l}\text { No one told } \\
\text { at home }\end{array}$ & $\begin{array}{l}\text { Teachers } \\
\text { not told }\end{array}$ \\
\hline Someone took personal belongings & $5240(77.6)$ & $1113(16.5)$ & $234(3.5)$ & $168(2.5)$ & 32.8 & 57.6 \\
\hline Someone threatened or blackmailed teenager & $6116(90.5)$ & $478(7.1)$ & $105(1.6)$ & $56(0.8)$ & 28.2 & 48.5 \\
\hline Someone hit or beat up teenager & $5984(88.6)$ & $582(8.6)$ & $120(1.8)$ & $68(1.0)$ & 24.8 & 41.0 \\
\hline Someone tricked teenager & $6234(92.2)$ & $446(6.6)$ & $46(0.7)$ & $35(0.5)$ & 30.2 & 54.5 \\
\hline Someone called teenager nasty names & $4319(64.0)$ & $1243(18.4)$ & $598(8.9)$ & $584(8.7)$ & 34.8 & 51.9 \\
\hline Peers would not hang around just to upset teenager & $6056(89.8)$ & $490(7.3)$ & $126(1.9)$ & $69(1.0)$ & 28.5 & 74.3 \\
\hline Peers tried to get teenager to do things he or she did not want to do & $6193(92.0)$ & $423(6.3)$ & $77(1.1)$ & $41(0.6)$ & 50.8 & 64.1 \\
\hline Peers told lies about teenager & $5608(83.7)$ & $765(11.4)$ & $221(3.3)$ & $105(1.6)$ & 34.3 & 66.5 \\
\hline Peers spoilt games to upset teenager & $6415(95.2)$ & $230(3.4)$ & $51(0.8)$ & $41(0.6)$ & 44.4 & 62.1 \\
\hline
\end{tabular}

Table 2 | Characteristics of complete case sample and overall Avon Longitudinal Study of Parents and Children (ALSPAC). Values are means (standard deviations) unless stated otherwise

\begin{tabular}{|c|c|c|c|c|}
\hline \multirow[b]{2}{*}{ Characteristics } & \multicolumn{2}{|l|}{ Samples } & \multirow[b]{2}{*}{$\begin{array}{l}\text { Total No } \\
\text { available }\end{array}$} & \multirow[b]{2}{*}{$P$ value } \\
\hline & $\begin{array}{l}\text { Complete case } \\
(n=2668)\end{array}$ & ALSPAC* & & \\
\hline No (\%) male & 45.5 & 53.1 & 13615 & $<0.001$ \\
\hline Emotional problems (10 years) & $1.48(1.40)$ & $1.68(1.50)$ & 8244 & $<0.001$ \\
\hline Conduct problems (10 years) & $1.49(1.65)$ & $1.52(1.68)$ & 8234 & 0.53 \\
\hline Concurrent bullying perpetration (13 years) & $0.81(1.69)$ & $0.97(2.11)$ & 6172 & 0.002 \\
\hline \multicolumn{5}{|l|}{ Family characteristics: } \\
\hline Lower parental social class (\%) & 40.4 & 57.8 & 10282 & $<0.001$ \\
\hline Maternal education O level or less (\%) & 48.4 & 66.6 & 11355 & $<0.001$ \\
\hline Mean (SD) maternal depression & $6.07(4.40)$ & $7.23(4.95)$ & 11.660 & $<0.001$ \\
\hline
\end{tabular}

$24-51 \%$ of teenagers reported that they never told their parents. Teenagers were most likely to report physical victimisation such as being hit or beaten up.

Table 2 shows the characteristics of our complete case sample versus the overall ALSPAC sample. Compared with the overall ALSPAC sample, participants retained in the complete case sample were more likely to be female and to have higher mean levels of emotional problems at age 10 years by maternal report. They were less likely to report concurrent perpetration of bullying and to have parents with lower social class or mothers with lower levels of education. Their mothers had fewer depressive symptoms compared with the ALSPAC sample. There were no differences between the complete case sample and the overall ALSPAC sample in concurrent depressive symptoms at age 13 years, in peer victimisation at age 13 years, or in depression at age 18 years.

Table 3 shows the sociodemographic and other characteristics of children who were never, occasionally, or frequently victimised at age 13 years. Compared with those who were not victimised, adolescents who were victimised by peers at age 13 years were more likely to be female and more likely to have displayed higher levels of emotional and behavioural problems before being bullied. Victimised adolescents were also more likely to display higher levels of concurrent depressive symptoms.

\section{Association with depression}

The proportion with depression increased with the frequency of victimisation (table 4). Just over 5\% of teenagers in the no victimisation group had depression increasing to nearly $15 \%$ in those reporting frequent victimisation. In terms of persistent depression, $10.1 \%$ of those who reported frequent victimisation said that they had experienced depressive symptoms for more than two years, compared with $4.1 \%$ of those in the non-victimised group. Despite a difference in overall prevalence rates between sexes, there was no evidence of an interaction between sex and peer victimisation $(P=0.45)$, and thus analyses were conducted for the whole sample and not stratified by sex. 


\begin{tabular}{|c|c|c|c|c|}
\hline Characteristics & $\begin{array}{l}\text { No victimisation } \\
(\mathrm{n}=3090)\end{array}$ & $\begin{array}{l}\text { Occasional } \\
\text { victimisation } \\
(n=2430)\end{array}$ & $\begin{array}{l}\text { Frequent } \\
\text { victimisation } \\
(\mathrm{n}=1199)\end{array}$ & $P$ value \\
\hline \multicolumn{5}{|l|}{ Individual characteristics: } \\
\hline Male (\%) & 50.6 & 47.3 & 47.6 & 0.02 \\
\hline Conduct problems & $1.1(1 \cdot 3)$ & $1.2(1 \cdot 3)$ & $1.5(1.6)$ & $<0.001$ \\
\hline Concurrent depressive symptoms (MFQ) & $1.0(0.7)$ & $1.4(0.7)$ & $1.9(0.7)$ & $<0.001$ \\
\hline Concurrent bullying perpetration (13 years) & $0.2(0.9)$ & $1.0(1.8)$ & $2.5(2.9)$ & $<0.001$ \\
\hline \multicolumn{5}{|l|}{ Family characteristics } \\
\hline Lower parental social class, (\%) & 47.7 & 44.6 & 46.0 & 0.12 \\
\hline
\end{tabular}

The increased rates of depression in those with frequent victimisation corresponded to an odds ratio of 2.96 (95\% confidence interval 2.21 to 3.97) compared with those who were not victimised. This association was reduced slightly when adjusting for confounding factors. The largest reduction in effect size was observed when adjusting for the effects of children's individual characteristics (sex, baseline emotional and behavioural problems, and concurrent depressive symptoms; odds ratio $2.14,42 \%$ reduction in effect size). Evidence of a dose-response relation was observed between the continuous measure of victimisation and depression (adjusted linear trend odds ratio 1.08, $\mathrm{P}=0.01)$. There was little evidence for a non-linear relation between victimisation and depression $(\mathrm{P}=0.07)$. We found no evidence of an interaction between depression and peer victimisation at 13 years in predicting depression at 18 years $(\mathrm{P}=0.34$, likelihood ratio test $\chi^{2}=2.19$ ), suggesting that victimisation is associated with both onset and persistence of depression. The population attributable fraction from the final multivariable logistic model suggested that 29.2\% (95\% confidence interval $10.9 \%$ to $43.7 \%$ ) of the total risk of depression at age 18 could be explained by peer victimisation in adolescence.

\section{Sensitivity analyses}

In additional sensitivity analyses we removed all participants with scores reaching clinical significance on the short moods and feelings questionnaire at age 13 (defined as scores of $\geq 11 ; n=473$ ). Our analyses were virtually unchanged: occasional victimisation 1.29 (0.87 to 1.92) and frequent victimisation 2.82 (1.86 to 4.26). Additionally, when the 87 participants who reported depressive symptoms for more than two years (according to the clinical interview schedule-revised) were excluded from the analyses, the results were only slightly attenuated: occasional victimisation 1.17 (0.85 to 1.60) and frequent victimisation 2.20 (1.54 to 3.14).

We also investigated whether mother reports of victimisation of their offspring during adolescence were associated with depression at age 18 years. Mother reports of victimisation at 12 years of age were associated with depression at age 18 years in unadjusted analyses $(1.38,1.10$ to 1.74$)$; however, there was no evidence for an association after adjusting for confounders (1.08, 0.78 to 1.49 ).

Owing to high amounts of missing data, we were unable to adjust for the potential confounding effects of paternal mental health in our multivariate models. In additional analyses, paternal depression and anxiety (assessed using the Crown Crisp experiential index) were associated with victimisation in offspring at age 13 ( $\beta=0.02$, 95\% confidence interval 0.01 to 0.03 , $\mathrm{P}<0.001)$ and depression in offspring $(1.02,1.00$ to 1.04 , $\mathrm{P}=0.05)$; however, neither attenuated the strength of the unadjusted relation by much (unadjusted odds ratio $1.13,95 \%$ confidence interval 1.09 to $1.17, \mathrm{P}<0.001$,

\begin{tabular}{|c|c|c|c|c|c|c|c|}
\hline \multirow[b]{2}{*}{$\begin{array}{l}\text { Victimisation } \\
\text { status }\end{array}$} & \multirow[b]{2}{*}{ No (\%) depressed } & \multicolumn{2}{|c|}{ Unadjusted odds ratio $(95 \% \mathrm{CI})$} & \multicolumn{4}{|l|}{ Odds ratio $(95 \% \mathrm{Cl})$} \\
\hline & & $\begin{array}{l}\text { All available data } \\
(\mathrm{n}=3898)\end{array}$ & $\begin{array}{l}\text { Complete cases } \\
(n=2668)\end{array}$ & $\begin{array}{l}\text { Adjusted } \\
(n=2668)\end{array}$ & $\begin{array}{l}\text { Unadjusted using } \\
\text { imputed dataset* } \\
(n=6472)\end{array}$ & $\begin{array}{l}\text { Adjusted using } \\
\text { imputed dataset } t \\
(n=3898)\end{array}$ & $\begin{array}{l}\text { Adjusted using } \\
\text { imputed dataset* } \\
(n=6472)\end{array}$ \\
\hline None & $1769(5.5)$ & 1.00 & 1.00 & 1.00 & 1.00 & 1.00 & 1.00 \\
\hline Occasional & $1446(7.1)$ & 1.31 (0.98 to 1.74$)$ & 1.34 (0.93 to 1.93$)$ & 1.08 (0.74 to 1.59$)$ & 1.35 (1.01 to 1.81$)$ & $1.08(0.80$ to 1.46$)$ & $1.13(1.02$ to 1.12$)$ \\
\hline Frequent & $683(14.8)$ & 2.96 (2.21 to 3.97$)$ & $3.33(2.32$ to 4.78$)$ & $2.32(1.49$ to 3.63$)$ & $2.82(2.05$ to 3.87$)$ & 2.00 (1.39 to 2.87$)$ & 1.87 (1.29 to 2.72$)$ \\
\hline Linear trend & - & $1.13(1.09$ to 1.17$)$ & 1.14 (1.09 to 1.18$)$ & 1.08 (1.02 to 1.14$)$ & 1.12 (1.08 to 1.16$)$ & 1.07 (1.03 to 1.12$)$ & $1.07(1.02$ to 1.12$)$ \\
\hline
\end{tabular}




\begin{tabular}{|c|c|c|c|c|}
\hline Victimisation status by age & No (\%) depressed & \multicolumn{3}{|l|}{ Odds ratio $(95 \% \mathrm{CI})$} \\
\hline \multicolumn{5}{|l|}{8 years: } \\
\hline None & $980(6.3)$ & 1.00 & 1.00 & 1.00 \\
\hline Frequent & 708 (11.7) & 2.02 (1.43 to 2.87$)$ & 1.91 (1.32 to 2.77$)$ & 1.38 (0.92 to 2.08) \\
\hline \multicolumn{5}{|l|}{10 years: } \\
\hline None & $1674(6.3)$ & 1.00 & 1.00 & 1.00 \\
\hline Occasional & $1510(8.2)$ & 1.31 (0.99 to 1.72) & 1.33 (0.98 to 1.80$)$ & 1.15 (0.84 to 1.57$)$ \\
\hline Frequent & $754(10.3)$ & 1.68 (1.24 to 2.29$)$ & 1.83 (1.30 to 2.58$)$ & 1.18 (0.80 to 1.73$)$ \\
\hline Frequent & $683(14.8)$ & 2.88 (2.14 to 3.87$)$ & 2.95 (2.13 to 4.08$)$ & 2.58 (1.81 to 3.67$)$ \\
\hline
\end{tabular}

adjusted for paternal depression and anxiety 1.12, 1.07 to $1.17, \mathrm{P}<0.001$ ).

\section{Missing data analyses}

Analyses were repeated using the imputed datasets (table 4). Results were consistent with the previous findings based on complete cases. The associations between overall victimisation score and depression were slightly attenuated-for example, the odds ratio for the fully adjusted model using the linear measure of victimisation was 1.08 (95\% confidence interval 1.02 to 1.14 ) compared with 1.07 (1.02 to 1.12 ) in the imputed dataset. However, the evidence of an association between victimisation and depression was still strong.

\section{Victimisation in adolescence versus childhood}

Overall rates of victimisation appeared to decline with age, with mean scores of 3.27 (SD 3.6) at age 8 years, 2.01 (SD 2.8) at age 10, and 1.82 (SD 2.8) at age 13. Correlations across age were modest, with polychoric correlation coefficients between 0.29 and 0.40. Despite the decrease in rates of victimisation, 255 new victims were identified at 13 years of age who had not reported victimisation at ages 8 and 10 years. The univariable results provide evidence for associations between victimisation in childhood (assessed at ages 8 and 10 years) and victimisation in adolescence with depression at age 18 years (table 5). There was still evidence of an association between victimisation at age 13 years and depression when all three exposure time points were included in the regression model.

\section{Discussion}

We found evidence for an association between victimisation by peers in adolescence and depression in young adulthood. There was a dose-response relation between frequency of peer victimisation in adolescence and the risk of developing depression meeting ICD-10 criteria at age 18 years. Adolescents who reported frequent bullying by peers were about twice as likely to develop depression compared with non-victimised peers, even after adjustment for previous depressive symptoms and previous victimisation and a range of other individual and family confounding factors. This association was seen in both males and females. The large population attributable fraction suggests that approximately $29 \%$ of the burden of depression at age 18 years could be attributed to victimisation by peers in adolescence if this were a causal relation. The longitudinal nature of our study reduces the possibility of reverse causality, whereas the impact of victimisation on depression has both clinical and biological plausibility. All these points support the hypothesis that this is a causal relation, although this is difficult to establish using observational data.

\section{Strengths and weaknesses of this study}

This study has several strengths. The large sample size and extended follow-up from early childhood to late adolescence spanned the period in which rates of depression increase rapidly and approach those found in adulthood. ${ }^{2}$ We had detailed self report measures of peer victimisation and adjusted for a large number of potential confounders, including previous victimisation. There is a concern that the victims might have characteristics that increase susceptibility to victimisation as well as independently increasing the risk of depression. With the wealth of previous data available in ALSPAC, our analyses found some evidence that this did occur, but even after adjustment for confounding there was still strong evidence for an association. Residual confounding cannot be ruled out but we think it unlikely that it could explain an association of this size.

A limitation of our study is the loss to follow up from the original ALSPAC sample. The young adults were more likely to attend the clinic if they came from families of a higher education and social class, indicating that data were not missing completely at random. Non-random response may therefore have biased our complete case analyses. However, the frequency of adolescent victimisation did not differ between the original ALSPAC sample and the complete case sample, and the 
results of analyses after multiple imputation led to some attenuation of the findings but were consistent with our complete case findings. The wealth of data on participants from previous assessments in ALSPAC allowed for imputation of missing data using a rich list of relevant variables. The extensive auxiliary information also ensures that the assumptions behind our imputation of missing data are much more reasonable. Even though we think it unlikely that such a strong association could be explained by attrition, selection bias remains a possibility.

A second limitation is that our principal measure of victimisation was self reported and people who are prone to depression may also be more likely to perceive or report incidences of victimisation. To tackle this, we adjusted the analysis for concurrent depressive symptoms and controlled for previous victimisation and emotional and behavioural problems. Furthermore, in additional sensitivity analyses we found that the association remained even after excluding participants who scored in the clinical range for depression at age 13 years. If anything this might have led to some over-adjustment if, as we would hypothesise, depression at an earlier age had resulted from earlier victimisation. The observed association also remained when we excluded participants who at age 18 years reported experiencing depression in the clinical range for two or more years. We did not have evidence to support an association between maternally reported victimisation and depression; however, many teenagers reported that they never told anyone at home about being victimised (table 1), suggesting that mothers may not be the best informants of peer victimisation at this age.

A third limitation is that we did not specifically ask about cyberbullying (that is, bullying that takes place through the use of electronic communication). Peer victimisation was assessed in 2003-05 at a time when there was less use of electronic media. The cross sectional strength of association between peer victimisation and later depressive symptoms in childhood has been reported to be similar for cyberbullying and other peer victimisation. Furthermore, most victims report experiencing both forms. ${ }^{32} 33$

Finally, it is not clear if our findings generalise beyond this UK population of children. Victimisation rates may vary from country to country, ${ }^{34}$ but peer victimisation seems to occur in all societies and we think the result of this study is likely to apply to many other societies. Furthermore, a consistent, graded association between victimisation and depressive symptoms has been observed using cross sectional data from 28 countries in Europe and North America. ${ }^{35}$ One study from the United States recently reported a relation between bullying in childhood and clinical depression in adulthood. ${ }^{36}$ We think it is likely that this result would also apply in many other societies, although such generalisability cannot be assured.

Finally, there are limitations inherent to observational data such as ours. For example, although we adjusted for several potential confounders, we cannot exclude the possibility of residual confounding.

\section{Victimisation in adolescence}

Our findings show that victimisation in adolescence is associated with depression irrespective of previous victimisation in childhood. Adolescence is characterised by rapid social, emotional, and physiological changes. Peer relationships increase in both importance and complexity, and self consciousness is heightened. It is a critical period for the development of social relationships outside of the home and could be a particularly sensitive time if these are disrupted. Although overall rates of peer victimisation are lower in adolescence compared with childhood, ${ }^{37}$ evidence suggests that peer victimisation may be more targeted and persistent in the teenage years. ${ }^{38}$ Animal studies using models of aversive social interactions during early adolescence have reported long lasting effects, enduring into adulthood. The mechanisms underlying this could include the monoamine projections that are implicated in some theories of depression. ${ }^{39}$ If such mechanisms are still relevant in humans, they might also be activated in teenagers exposed to victimisation and contribute to the vulnerability to future stressors and the development of depressive symptoms. Relatively few studies have examined mediating mechanisms underlying the association between peer victimisation and depression, and it is likely that both psychosocial and biological factors play a role. Studies have suggested a potential role for perception of threat and sense of control, 40 emotion dysregulation, ${ }^{41}$ blunted cortisol reactivity, ${ }^{42}$ and increased methylation of the serotonin transporter gene ${ }^{43}$ among others.

\section{Implications for research, practice, and policy}

This study is one of the largest to date to investigate whether victimisation by peers in adolescence is associated with the emergence of clinical depression, and has dealt with some of the limitations of previous longitudinal studies. Our findings support the hypothesis that victimisation in adolescence can cause depression and could make a substantial contribution to the burden of depression in adulthood. In particular we were able to adjust for several factors that might have caused both victimisation and depression. These results accord with clinical experience in which many adults with depression report periods of bullying during adolescence. Also of relevance are the reports of suicide and self harm among adolescents attributed to victimisation, a topic of much public concern and media interest. ${ }^{4-46}$ Our findings also highlight that a lot of victimisation may go unreported, with many adolescents reporting that they tell no one either at home or at school.

Depression is a major public health problem worldwide, with high social and economic costs. If peer victimisation in adolescence is a causal factor then the prevention of victimisation in schools could be an effective means of reducing the incidence of depression. School based programmes based on cognitive behavioural therapy have been disappointing. ${ }^{3}$ Although there is evidence that consistent antibullying measures in schools can reduce the rates of victimisation, ${ }^{47}$ 
our findings suggest that such interventions during adolescence could help to reduce the burden of depression later in life.

\section{Research in context: systematic review}

We searched PubMed for original research published in any year that prospectively investigated the impact of peer victimisation on depression meeting diagnostic criteria in late adolescence or adulthood (defined as age $\geq 18$ years, using the terms "bullying", "peer victimisation", and "depression"). Most of the research in this area has focused on the short term associations between peer victimisation and depressive symptoms in childhood and was excluded. Few studies of peer victimisation used a clinically recognised measure of depression in late adolescence or adulthood. We identified only six relevant papers. Of these, one had not adjusted for key confounders, including baseline depressive symptoms, ${ }^{13}$ and two others had not adjusted for earlier behavioural problems ${ }^{1448}$ that might themselves have led to both victimisation and later depression. Two other studies ${ }^{15}{ }^{16}$ have only found statistical evidence for an association for children who are both the victims and the perpetrators of bullying. Perpetrators also display high levels of conduct problems and reactive aggression, which are themselves associated with later depression. ${ }^{1718}$. Finally, several studies relied on a single-item measure of peer victimisation. ${ }^{14-16}{ }^{19}$ Given the limitations of the studies identified in our literature search, it remains unclear whether peer victimisation in adolescence contributes to the overall public health burden of clinical depression.

\section{Interpretation}

Our study is one of the largest to date to examine whether peer victimisation is prospectively associated with depression meeting diagnostic criteria in the clinically relevant range. The main strength of this study is the wide range of confounders and comprehensive measure of peer victimisation. We observed a strong, graded association of peer victimisation in adolescence with depression meeting diagnostic criteria at age 18 years, independent of the effects of the experiences of victimisation in childhood, baseline emotional and behavioural problems, and a range of other key confounders. Our findings suggest that approximately $29 \%$ of the burden of depression at age 18 years could be attributed to peer victimisation. These findings lead us to conclude that peer victimisation during adolescence may contribute significantly to the overall public health burden of clinical depression and that intervention to reduce peer victimisation in secondary schools should reduce the burden of depression.

We thank the families who took part in this study, the midwives for help in recruiting them, and the ALSPAC team, which includes interviewers, computer and laboratory technicians, clerical workers, research scientists, volunteers, managers, receptionists, and nurses.

Contributors: LB and GL conceived and designed the study, with advice from CJ and DW. LB analysed the data, and LB, CJ, DW and GL interpreted the data. LB drafted the manuscript. CJ, DW, and GL criticised the manuscript for important intellectual content. All authors have read and approved the final version of the manuscript. This article is the work of the authors. LB serves as guarantor for the contents of this article. All authors, external and internal, had full access to all of the data (including statistical reports and tables) in the study and can take responsibility for the integrity of the data and the accuracy of the data analysis. All researchers are independent of the funding bodies.

Funding: The UK Medical Research Council and the Wellcome Trust (grant reference 092731) and the University of Bristol provide core support for ALSPAC. This research was specifically funded by a Wellcome Trust grant held by GL (grant reference 084268/Z/07/Z). LB was partly supported by a grant from the Jacobs Foundation, and is supported by a Leverhulme Trust Early Career Fellowship; DW was partly supported by grant ES/K003593/1 of the Economic and Social Research Council.

Competing interests: All authors have completed the ICMJE uniform disclosure form at www.icmje.org/coi_disclosure.pdf and declare: no financial relationships with any organisations that might have an interest in the submitted work in the previous three years, and no other relationships or activities that could appear to have influenced the submitted work.

Ethical approval: This study was approved by the ALSPAC Law and Ethics Committee and the local research ethics committees.

Data sharing: The ALSPAC policy on data sharing is available at www. bristol.ac.uk/alspac. To discuss access to ALSPAC data, please contact the ALSPAC executive team at alspac-exec@bristol.ac.uk.

Transparency: The lead author (LB) affirms that the manuscript is an honest, accurate, and transparent account of the study being reported; that no important aspects of the study have been omitted; and that any discrepancies from the study as planned have been explained.

This is an Open Access article distributed in accordance with the terms of the Creative Commons Attribution (CC BY 4.0) license, which permits others to distribute, remix, adapt and build upon this work, for commercial use, provided the original work is properly cited. See: http://creativecommons.org/licenses/by/4.0/

1 World Health Organization. Global burden of disease: 2004 update. WHO, 2008. www.who.int/topics/global_burden_of_ disease/en/.

2 Jaffee SR, Moffitt TE, Caspi A, et al. Influence of adult domestic violence on children's internalizing and externalizing problems: an environmentally informative twin study. J Am Acad Child Adolesc Psychiatry 2002;41:1095-103.

3 Stallard P, Sayal K, Phillips R, Taylor JA, et al. A classroom based cognitive behavioural therapy in reducing symptoms of depression in high risk adolescents: pragmatic cluster randomised controlled trial. BMJ 2012;345

4 Prinstein M, Aikins J. Cognitive moderators of the longitudinal association between peer rejection and adolescent depressive symptoms. J Abnorm Child Psychol 2004;32:147-58.

5 Hawker DS, Boulton MJ. Twenty years' research on peer victimization and psychosocial maladjustment: a meta-analytic review of cross-sectional studies. / Child Psychol Psychiatry 2000;41:441-55.

6 Kaltiala-Heino R, Rimpela M, Marttunen M, et al. Bullying, depression, and suicidal ideation in Finnish adolescents: school survey. BMJ 1999;319:348-51.

7 Meltzer H, Vostanis P, Ford T, et al. Victims of bullying in childhood and suicide attempts in adulthood. Eur Psychiatry 2011;26:498-503.

8 Arseneault L, Milne BJ, Taylor A, et al. Being bullied as an environmentally mediated contributing factor to children's internalizing problems: a study of twins discordant for victimization. Arch Pediatr Adolesc Med 2008;162:145-50.

9 Kochenderfer-Ladd B, Wardrop IL. Chronicity and instability of children's peer victimization experiences as predictors of loneliness and social satisfaction trajectories. Child Develop 2001;72:134-51.

10 Zwierzynska K, Wolke D, Lereya T. Peer victimization in childhood and internalizing problems in adolescence: a prospective longitudinal study. J Abnorm Child Psychol 2012:1-15.

11 Bond L, Carlin JB, Thomas L, et al. Does bullying cause emotional problems? A prospective study of young teenagers. BM 2001;323:480-4

12 Brunstein Klomek A, Kleinman M, Altschuler E, et al. NIH public access. Suicide Life Threat Behav 2012;41:501-16.

13 Gibb SJ, Horwood LJ, Ferguson D. Bullying victimization/perpetration in childhood and later adjustment: findings from a 30 year longitudinal study. J Aggress Confl Peace Res 2011:3·82-8.

14 Kaltiala-Heino R, Frojd S, Marttunen M. Involvement in bullying and depression in a 2-year follow-up in middle adolescence. Eur Child Adolesc Psychiatry 2010;19:45-55.

15 Klomek AB, Sourander A, Kumpulainen K, et al. Childhood bullying as a risk for later depression and suicidal ideation among Finnish males. J Affect Disord 2008;109:47-55. 
16 Copeland WE, Wolke D, Angold A, et al. Adult psychiatric outcomes of bullying and being bullied by peers in childhood and adolescence. JAMA Psychiatry 2013:1-8.

17 Bowes L, Arseneault L, Maughan B, et al. School, neighborhood and family factors are associated with children's bullying involvement: a nationally-representative longitudinal study. J Am Acad Child Adolesc Psychiatry 2009; 48:545-53.

18 Stringaris A, Lewis G, Maughan B. Developmental pathways from childhood conduct problems to early adult depression: findings from the ALSPAC cohort. Br / Psychiatry 2014:205:17-23.

19 Takizawa R, Maughan B, Arseneault L. Adult health outcomes of childhood bullying victimization: evidence from a five-decade longitudinal British birth cohort. Am J Psychiatry 2014:171:777-84.

20 Golding J, Pembrey M, Jones R. ALSPAC-the Avon Longitudinal Study of Parents and Children. I. study methodology. Paediatr Perinat Epidemiol 2001;15:74-87.

21 Boyd A, Golding J, Macleod J, et al. Cohort profile: the "children of the 90s'-the index offspring of the Avon Longitudinal Study of Parents and Children. Int J Epidemiol 2013;42:111-27.

22 Lewis G. Assessing psychiatric disorder with a human interviewer or a computer. J Epidemiol Community Health 1994;48:207-10.

23 Wolke D, Woods S, Stanford K, et al. Bullying and victimization of primary school children in England and Germany: prevalence and school factors. BritJ Psychol 2001;92:673-96.

24 Stapinski LA, Bowes L, Wolke D, et al. Peer victimization during adolescence and risk for anxiety disorders in adulthood: a prospective cohort study. Depress Anxiety 2014;31:574-82.

25 Goodman R. Psychometric properties of the Strengths and Difficulties Questionnaire. J Am Acad Child Adolesc Psychiatry 2001;40:1337-45.

26 Shakoor S, Jaffee S, Andreou P, et al. Mothers and children as informants of bullying victimisation: results from an epidemiological cohort of children. J Abnorm Child Psychol 2011;39:379-87.

27 Angold A, Costello EJ, Messer SC, et al. The development of a short questionnaire for use in epidemiological studies of depression in children and adolescents. Int J Method Psych 1995;5:237-49.

28 Dale A, Marsh C. The 1991 census user's guide. Office of National Statistics, 1993.

29 Cox JL, Holden JM, Sagovsky R. Detection of postnatal depression. Development of the 10-item Edinburgh Postnatal Depression Scale. BrJ Psychiatry 1987;150:782-6.

30 White I, Royston P, Wood AM. Multiple imputation using chained equations: issues and guidance for practice. Stat Med 2011;30:377-99.

31 Little RJA, Rubin DB. Statistical analysis with missing data. 2nd ed. Wiley, 2002.

32 Beckman L, Hagquist C, Hellström L. Does the association with psychosomatic health problems differ between cyberbullying and traditional bullying? Emot Behav Diff 2012;17:421-34
33 Olweus D. Cyberbullying: an overrated phenomenon?. Eur J Dev Psychol 2012;9:520-38.

34 Nansel TR, Craig W, Overpeck MD, et al. Cross-national consistency in the relationship between bullying behaviors and psychosocial adjustment. Arch Pediatr Adolesc Med 2004;158:730-6.

35 Due P, Holstein BE, Lynch J, et al. Bullying and symptoms among school-aged children: international comparative cross sectional study in 28 countries. Eur J Public Health 2005;15:128-32.

36 Copeland WE, Wolke D, Angold A, et al. Adult psychiatric outcomes of bullying and being bullied by peers in childhood and adolescence. JAMA Psychiatry 2013;70:419-26.

37 Smith PK, Madsen K, Moody J. What causes the age decline in being bullied at school? Towards a developmental analysis of risks of being bullied. Educ Res 1999:41:267-85.

38 Boulton MJ, Smith PK. Bully/victim problems in middle-school children: stability, self-perceived competence, peer perceptions and peer acceptance. Br / Dev Psychol 1994:12:315-29.

39 Bingham B, McFadden K, Zhang X, et al. Early adolescence as a critical window during which social stress distinctly alters behavior and brain norepinephrine activity. Neuropsychopharmacology 2011;36:896-909.

40 Hunter SC, Durkin K, Heim D, et al. Psychosocial mediators and moderators of the effect of peer-victimization upon depressive symptomatology. J Child Psychol Psychiatry 2010;51:1141-9.

41 McLaughlin KA, Hatzenbuehler ML, Hilt LM. Emotion dysregulation as a mechanism linking peer victimization to internalizing symptoms in adolescents. J Consult Clin Psychol 2009;77:894

42 Ouellet-Morin I, Odgers CL, Danese A, et al. Blunted cortisol responses to stress signal social and behavioral problems among maltreated/ bullied 12-year-old children. Biol Psychiatry 2011;70:1016-23.

43 Ouellet-Morin I, Wong CC, Danese A, et al Increased serotonin transporter gene (SERT) DNA methylation is associated with bullying victimization and blunted cortisol response to stress in childhood: a longitudinal study of discordant monozygotic twins. Psychol Med 2013;43:1813-23.

44 BBC News. 2014. Bullied teenager left goodbye message, mother says. www.bbc.co.uk/news/uk-wales-south-east-wales-26873918.

45 BBC News. 2013. Shy Keenan's son “kept overdose drugs at Philip Morant school." www.bbc.co.uk/news/uk-england-essex-24959371.

46 BBC News. Facebook bullying suicide boy's parents in law change call. 2011. www.bbc.co.uk/news/uk-england-birmingham-14121631.

47 Ttofi MM, Farrington DP. Effectiveness of school-based programs to reduce bullying: a systematic and meta-analytic review. J Exp Criminol 2011;7:27-56.

48 Schwartz D, Lansford JE, Dodge KA, et al. Peer victimization during middle childhood as a lead indicator of internalizing problems and diagnostic outcomes in late adolescence. J Clin Child Adolesc Psychol 2015 May-Jun;44:393-404.

(C) BMJ Publishing Group Ltd 2015 